Han de Vries · Jacobus C. Biesmeijer

\title{
Self-organization in collective honeybee foraging: emergence of symmetry breaking, cross inhibition and equal harvest-rate distribution
}

Received: 16 July 2001 / Revised: 30 December 2001 / Accepted: 12 January 2002 / Published online: 27 March 2002

(C) Springer-Verlag 2002

\begin{abstract}
De Vries and Biesmeijer described in 1998 an individual-oriented model that simulates the collective foraging behaviour of a colony of honeybees. Here we report how this model has been expanded and show how, through self-organization, three colony-level phenomena can emerge: symmetry breaking, cross inhibition and the equal harvest-rate distribution. Symmetry breaking is the phenomenon that the numbers of foragers visiting two equally profitable food sources will diverge after some time. Cross inhibition is the phenomenon that, by increasing the profitability of one of two equal food sources, the number of foragers visiting the other source will decrease. In some circumstances, the bees foraging on two sources of different profitabilities will be distributed between these sources such that the two average energy harvest rates are equal. We will refer to this phenomenon as the equal harvest-rate distribution. For each of these three phenomena, we show what the necessary behavioural rules to be followed by the individual forager bees are, and what the necessary circumstances are (that is, what values the model parameters should take) in order for these phenomena to arise. It seems that patch size and forager group size largely determine when each of these phenomena will arise. Experimenting with two types of currency, net gain rate and net gain efficiency, revealed that only gain rate may result in an equal harvest-rate distribution of foragers visiting different food sources.
\end{abstract}

Keywords Honeybee Collective foraging · Communication · Individual-oriented model . Self-organization

Communicated by R.F.A. Moritz

H. de Vries $(\bowtie) \cdot$ J.C. Biesmeijer

Department of Behavioural Biology, Utrecht University, P.O. Box 80.086, 3508 TB Utrecht, The Netherlands e-mail: J.deVries@bio.uu.nl

Fax: +31-30-2521105

\section{Introduction}

Many patterns and structures found in social insect colonies emerge through self-organization (Bonabeau et al. 1997; Detrain et al. 1999). The individual workers use only local information. They do not follow instructions put forward by some central organizer or leader; they do not have blueprints, recipes or templates of the pattern to be built; rather, the emerging structure or pattern results from the multitude of individual actions (Camazine et al. 2001). In honeybees, for example, self-organization gives rise to thermoregulation (Watmough and Camazine 1995), circadian rhythms (Moritz and Kryger 1994), collective defensive behaviour (Millor et al. 1999), nectar source selection (Camazine and Sneyd 1991; de Vries and Biesmeijer 1998), nectar collecting and processing (Seeley 1995; Anderson 1998; Anderson and Ratnieks 1999) and nest-site selection (Seeley and Buhrman 1999; Visscher and Camazine 1999). The many feedback loops and large number of interactions make that the dynamics of self-organized systems are sometimes non-intuitive and hard to predict. Therefore, individual-oriented modelling and related approaches provide powerful tools to understand such systems.

In an earlier paper, de Vries and Biesmeijer (1998) presented an individual-oriented simulation model that could reproduce successfully the collective forager patterns of a honeybee colony, visiting two feeders, each at $400 \mathrm{~m}$ distance from the hive as observed in the field by Seeley et al. (1991). An effort was made to include those individual behavioural rules that are necessary and sufficient for generating the observed collective foraging patterns. Special attention was given to the empirical validity of the behavioural rules themselves and questions pertaining to this aspect that arose during the modelling. We believe that one of the main advantages of individualoriented modelling is that it makes possible a stronger coupling between the two phases in scientific research: obtaining empirical data and constructing an explanatory theoretical model. Another advantage of combining individual-oriented simulation modelling with empirical re- 
search is that it helps the researcher to find adequate concepts necessary for understanding the observed phenomena (see Biesmeijer and de Vries 2001).

The main results obtained by experimenting with our former model are that a good fit between observed and simulated collective forager patterns was obtained when the model included rules in which the bees: (1) relied on the information acquired from previous flights to a source (e.g. profitability and time of the day when the source was found), (2) used positional information obtained by attending recruitment dances (waggle dances), and (3) did not abandon a (temporarily) deteriorated source too fast or too slowly.

In the current paper, we present further developments of our simulation model. First, we try to pay full attention to the empirical validity of the new behavioural rules included in the model; and second, we investigate how, through self-organization, colony-level forager allocation patterns emerge from the individual actions. The earlier model has been slightly changed and expanded in several repects. Using this expanded model, we were able to simulate three colony-level foraging patterns: symmetry breaking, cross inhibition and the emergence of an equal harvest-rate distribution. Symmetry breaking may occur when a colony starts foraging on two equally valuable sources, but after some time the forager stream to one source outcompetes the stream to the other source. This phenomenon has been observed in ants by Pasteels et al. (1987) but, as far as we know, not yet in honeybees. In their review paper on self-organization in insect societies, Bonabeau et al. (1997), when discussing foraging in bees, state that "If the colony is offered two identical food sources at the same distance from the nest, the bees exploit the two sources symmetrically". Symmetry breaking occurs when the positive feedback process regulating the one stream of foragers accidently becomes slightly faster than the other one, or when the negative feedback process becomes slightly slower, or through some combination of these. The phenomenon of cross inhibition occurs when the value of one of two equally valuable sources is increased, with the subsequent result that the stream of foragers to the other source diminishes, although its value has not been changed. This phenomenon has been observed by Seeley (1995, p 142) and is due to a longer search time for nectar-receiving bees in the hive by foragers returning home with a nectar load from the unchanged food source. Finally, we will show that if the size of food patches is not too large, the foraging bees of a colony will distribute themselves among the patches according to an equal harvest-rate distribution: that is, the average per capita gain rate is the same for both sources.

Our main objective of developing this individual-oriented model is to discover the behavioural rules followed by forager bees, and thereby improve our understanding of the adaptive collective behaviour shown by honeybee colonies during foraging. All three colony-level phenomena emerge through self-organization from the interlocked actions of many individual honeybee foragers, both in the field and in the hive. By simulating faithfully the individual-level behavioural rules that give rise to these phenomena, our model can generate a wide range of collective foraging behaviours of honeybee colonies, and may become a valuable tool for predicting under what circumstances new collective patterns can arise.

\section{Methods}

Modelling and simulation

In an earlier paper (de Vries and Biesmeijer 1998), we introduced an individual-oriented model constructed as a SMALLTALK simulation program, using the Hobo event-driven simulation system developed by Lhotka (1994). Individual-oriented (or individualbased) modelling has been described by Hogeweg and Hesper (1990) and Judson (1994). We refer the reader to our previous paper for all details concerning the model bee, the model environment, the input parameters for the model and the model behavioural rules. The nucleus of our individual-oriented model is the behaviour control structure (BCS) of a forager bee (see Fig. 2 in our earlier paper). Such a BCS will be a valid representation of a nectar forager in general if each particular foraging history of an arbitrary forager can be derived from it. In other words, a valid BCS means that all possible foraging histories are implicit in this BCS.

Our original model is extended here with four extra rules, and one rule has been modified. The modified rule is the following one (compare Fig. 1 in de Vries and Biesmeijer 1998). When a bee is loading nectar at a source, the time for collecting a load increases with increasing sugar concentration. In our earlier model we had: decreases with increasing sugar concentration. In his paper of 1994, Seeley writes (Table 1): "The "at feeder" and "at hive" times differ markedly between the near $(1.5 \mathrm{M})$ and far-feeder $(2.5 \mathrm{M})$ bees, evidently because of the strong difference in viscosity of their sugar solutions, which influences the time required to load and unload the solutions". This was overlooked by us. This minor change hardly changes the results presented in our former paper (see Results below), because the loading time represents only a small part of the total trip time, which is one of the relevant factors influencing the bee's subsequent behaviour. In the sequel, we will call this slightly modified model the basic model.

Much more important than this modification are the behavioural rules we have added. The following extensions have been added to the basic model to enable simulating a greater range of phenomena.

\section{Additional rule 1: finding a receiver bee and tremble dancing}

A bee that returns to the nest with a load of nectar has to find a receiver bee to whom she can transfer her load. In the situation that there is a constant number of bees potentially available as receiver bee, this search time depends on the number of bees that are simultaneously busy with transferring their load to a receiver bee (Seeley and Tovey 1994). This has been modelled by letting the nectar-loaded bee, upon returning to the nest, check in her local environment for the presence of an idle receiver bee. If no idle receiver bee is found, unloading is not possible for her at that moment. Subsequently, she will check regularly (every $12 \mathrm{~s}$ up to maximally $3 \mathrm{~min}$ ) whether there is a free receiver bee in her neighbourhood. If so, she will unload her nectar. We modelled the availability of receiver bees and the search time for an idle one in the following way. We took the value of 100 idle receiver bees as representing the situation in which a forager bee does not experience any delay in finding a receiver bee. When a smaller number, say $R$, of idle receiver bees is present, the chance for a forager bee to find an idle receiver bee is $R / 100$. As described above, every $12 \mathrm{~s}$ a forager bee tries to detect an idle receiver bee. This means 
that when, at a certain moment, there are 50 idle receiver bees, the chance for a forager bee that she will find 1 of these at $1 \mathrm{~min}$ after she started searching is $0.5^{5}=0.031$. The chance that she has already found a receiver bee before this moment is 0.9375 . When a forager has found an idle receiver bee, $R$ is decreased by 1 during transference of the nectar. The time to store the nectar by the receiver bee has not been modelled. The time till the forager has found a free receiver bee subsequently regulates the occurrence of three other behaviours of this forager.

First, the chance that she will subsequently start waggle dancing depends on this time till transference of nectar (and on the profitability of her source as well). This dependency of the probability of waggle dancing on the search time has been implemented in our model in conformity with the results presented by Seeley (1992, Fig. 7). The probability of waggle dancing decreases approximately linearly from 1 to 0 when the search time increases from 0 to $60 \mathrm{~s}$ for a bee that brings in food from a rich source containing $2.5 \mathrm{M}$ sugar solution. For nectar from a poor source containing a 1.25 -M sugar solution, we have set this probability twice as low, which means that after a search time of $30 \mathrm{~s}$, such a forager will not perform any waggle dancing anymore.

When the bee does not perform a waggle dance, she either starts tremble dancing or will not dance at all (Seeley 1992). By tremble dancing, she will recruit additional bees to function as receiver bees (Seeley et al. 1996). When a tremble dance is performed, one extra receiver bee is added to the available receiver bees. The probability for a bee that has visited a rich $2.5-\mathrm{M}$ nectar source to start tremble dancing increases linearly from 0 to 1 when her search time increases from 20 to $80 \mathrm{~s}$. For a bee that has visited a poor source containing $1.25-\mathrm{M}$ sugar, this probability is set to 5 times less (cf. Seeley 1992, Fig. 8).

Finally, we hypothesized that there is also a probability for the bee to abandon her source when the search time for a receiver bee is long. The abandoning probability for a bee that has visited a rich 2.5-M source increases from 0 to 1 when the search time increases from $30 \mathrm{~s}$ to $180 \mathrm{~s}$. For a bee that has visited a poor $1.25-\mathrm{M}$ source, this probability is set twice as high.

\section{Additional rule 2: waiting at the nectar patch}

Besides this search time (waiting time) at the unloading side of the foraging process, we also included the possibility of a waiting time at the loading side. Specifically, we introduced a patch size for a nectar source, which we define as the number of bees that can simultaneously imbibe nectar from it. When all available places are occupied, a bee has to fly around and wait until a place will become free at this source. If, by flying around, the total distance flown by a bee after leaving the nest exceeds a certain value (in our model set arbitrarily to a fixed value of 1,000 m), this bee will return to the nest empty.

In the experiment performed by Seeley and his co-workers (Seeley et al. 1991), which we simulated in our earlier model (de Vries and Biesmeijer 1998), the experimental feeder provided space to about 20 bees for simultaneously imbibing nectar (Seeley 1995, pp 76, 77; von Frisch 1967, pp 18-20). Most of our simulation runs were done with patch sizes of $5,10,20$ and 40 bees.

\section{Additional rule 3: behaviours depending on energy gains and costs}

In our earlier paper, we used as criterion of source profitability simply the sugar concentration (cf. Seeley et al. 1991). In the current model, we implemented a profitability criterion that not only involves the energetic gains obtained per foraging trip but also the energetic costs made. Specifically, following Seeley (1994), we divided the feeding trip into four segments: flight out, at feeder, flight in and at hive. For each of these four segments, the time was recorded and the corresponding energy-cost expenditure calculated according to the formulas presented in Seeley (1994). Subsequently, the probability for starting a waggle dance and the duration of the waggle dance are made dependent on the energy effi- ciency, (gain-cost)/cost, or, alternatively, on the energy rate, (gaincost)/time, where time is the total trip time. The equations linking either profitability criterion to the dance probability and duration have been devised such that they are compatible with the original equations linking the sugar concentration of the source to dance probability and duration.

For the dependency of waggle dancing on energetic efficiency, we constructed the following equations: Prob(waggle dance)= $0.0435^{*}$ (gain-cost)/cost -0.74 . This means that for an efficiency below $17 \mathrm{~J} / \mathrm{J}$, the bee will not start to dance. Starting from an efficiency of $17 \mathrm{~J} / \mathrm{J}$, the probability of dancing increases linearly to 1.0 when the efficiency rises to $40 \mathrm{~J} / \mathrm{J}$. For the dance duration we used the same equation: duration of waggle dance (in minutes $)=0.0435 *($ gain - cost $) /$ cost -0.74 .

For the dependency of waggle dancing on net energy rate, we constructed the following equations: Prob(waggle dance)= $0.66 *$ (gain-cost)/time- 0.32 . This means that below a net energy rate of $0.5 \mathrm{~J} / \mathrm{s}$ the bee will not start to dance. When the net energy rate increases to $2.0 \mathrm{~J} / \mathrm{s}$, the dance probability increases linearly to 1.0. For the dance duration, we used the same equation: duration of waggle dance (in minutes) $=0.66 *$ (gain-cost)/time-0.32.

The probability of flying out again to her known source also depends on the source profitability, either the energetic efficiency or net energy rate.

\section{Additional rule 4: only one bee can follow a waggle dance}

A fourth extension (or rather modification) concerns the number of bees that can simultaneously follow a dancing bee. In the earlier model, several bees could simultaneously attend to the same dancing bee, extract directional and distance information, and subsequently fly out. Following the detailed observations made by Judd (1995), we decided to change the model in such a way that at most one bee at a time can follow the dance (for some uniformly distributed random time between 6 and $18 \mathrm{~s}$ ) and extract information from it. In his paper, Judd stated: "It appears that although the average number of bees simultaneously orienting to a dancer can be as high as five or six (see Fig. 52 of von Frisch 1967), at most only one or two bees are simultaneously receiving the dance information".

This modified rule damps the per capita recruitment rate as compared to the earlier model. It does not change in a significant way the overall forager patterns (as is shown in the Results section).

\section{Results}

Simulation of 2-day experiment of Seeley et al. (1991) and role of patch size

Before presenting our simulation results with respect to the new collective phenomena, we first have to examine the possible effects of the changes in the bee rules on the simulation results of the 2-day experiment originally performed by Seeley et al. (1991), which we used as a testbed for developing our model (de Vries and Biesmeijer 1998). We were also interested to find out how the forager pattern (i.e. the numbers of foragers per half-hour visiting the two sources) is influenced by the size of the food patches. In particular, we wanted to check whether we could reproduce our earlier results with this extended model.

The results presented in Fig. 1 show that the forager pattern obtained by Seeley et al. (1991) in their experiments can emerge in simulation runs using this more ex- 

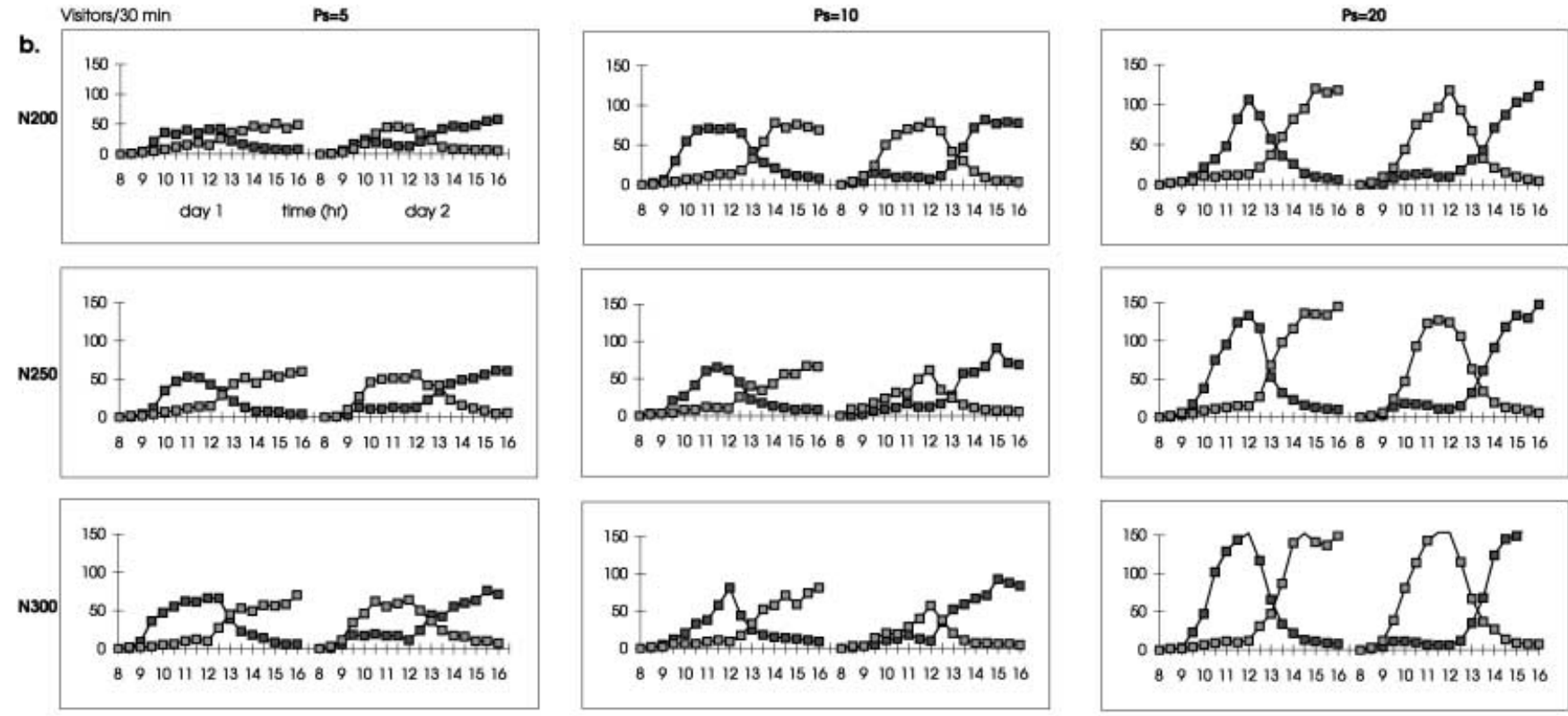

Fig. 1 a Forager pattern as observed by Seeley et al. (1991) in their 2-day experiment with two fluctuating sugar solution sources. In each graph, the values along the vertical axis indicate the number of different foragers visiting each of the sources during the previous half-hour (similarly in Figs. 2, 3, 4). b Results of simulation runs showing adaptive allocation of foragers to two sources containing changing concentrations of sugar solution. Nine simulated 2-day patterns of foragers visiting 2 nectar sources at $400 \mathrm{~m}$ north and south from the nest, with different values of forager work size $(N=200,250,300)$ and different patch sizes for both nectar sources $(P s=5,10,20)$. Each dot represents the number of different foragers visiting a source during half an hour. In all runs the sugar concentrations in the sources are varied over time in accordance with the original experiment performed by Seeley et al. (1991). Source 1 (black dots): day 1, 8-12 h: $2.5 \mathrm{M} ; 12-16 \mathrm{~h}$ : $0.75 \mathrm{M}$; day $2,8-12 \mathrm{~h}: 0.75 \mathrm{M} ; 12-16 \mathrm{~h}: 2.5 \mathrm{M}$. Source 2 (white dots): day 1,8-12 h: $1.0 \mathrm{M} ; 12-16 \mathrm{~h}: 2.5 \mathrm{M}$; day 2, 8-12 h: $2.5 \mathrm{M}$; $12-16 \mathrm{~h}: 0.75 \mathrm{M}$. The number of bees initially committed (Ic) to each source is 10 . Other parameters are set to standard values: transmission accuracy $=0.85$, search ability $=8$, abandoning probability $=0.07$

tended model as well. So, this modified and extended model is still capable of faithfully reproducing our earlier simulation results, involving the adaptive allocation of foragers to two sources containing time-varied concentrations of sugar solution. This validation of our new model with respect to earlier results is required since we are striving towards a model that contains a set of rules which are necessary and sufficient for simulating an increasing set of phenomena. The following sections contain simulation results of these new phenomena. In all following simulation runs, the values of the three parameters controlling information-processing characteristics of the individual bees have been set to their standard values: that is, transmission accuracy $\mathrm{t}=0.85$, search ability $\underline{\mathrm{s}}=8$ and abandoning probability $\underline{\mathrm{a}}=0.07$ (see de Vries and Biesmeijer 1998 for an explanation of these parameters). In our earlier model, we took as standard value for $\underline{s}$ a value of 4 ; in the present model, we have set $\underline{s}$ to 8 as standard value to offset the somewhat less severe recruitment rate caused by the introduction of rule 4 .

\section{Symmetry breaking}

Symmetry breaking is the phenomenon that the numbers of bees of a colony foraging on two equally valuable sources, diverge. This phenomenon has hardly been investigated in honeybees. Seeley and Levien (cited in Pasteels et al. 1987, p 171) reported the results of an experiment with two identical sources, located on opposite sides of the nest, in which the bees happened to distribute themselves equally across the sources. More recently, Bonabeau et al. (1997, p 190) also stated that "If the colony is offered two identical food sources at the same distance from the nest, the bees exploit the two sources symmetrically".

Symmetry breaking occurs if, at some moment, the number of bees exploiting one source starts rising faster than for the other source. To gain insight into the circumstances that bring about the occurrence of symmetry breaking, we varied the size of the forager pool $(N)$, the number of bees that are initially exploiting the sources [that is, the bees that are initially committed (Ic) to either source], and the size of the food patches (Ps).

We simulated a situation in which the returning forager bees did not experience any search time for finding a receiver bee. These runs simulated $960 \mathrm{~min}$. The 

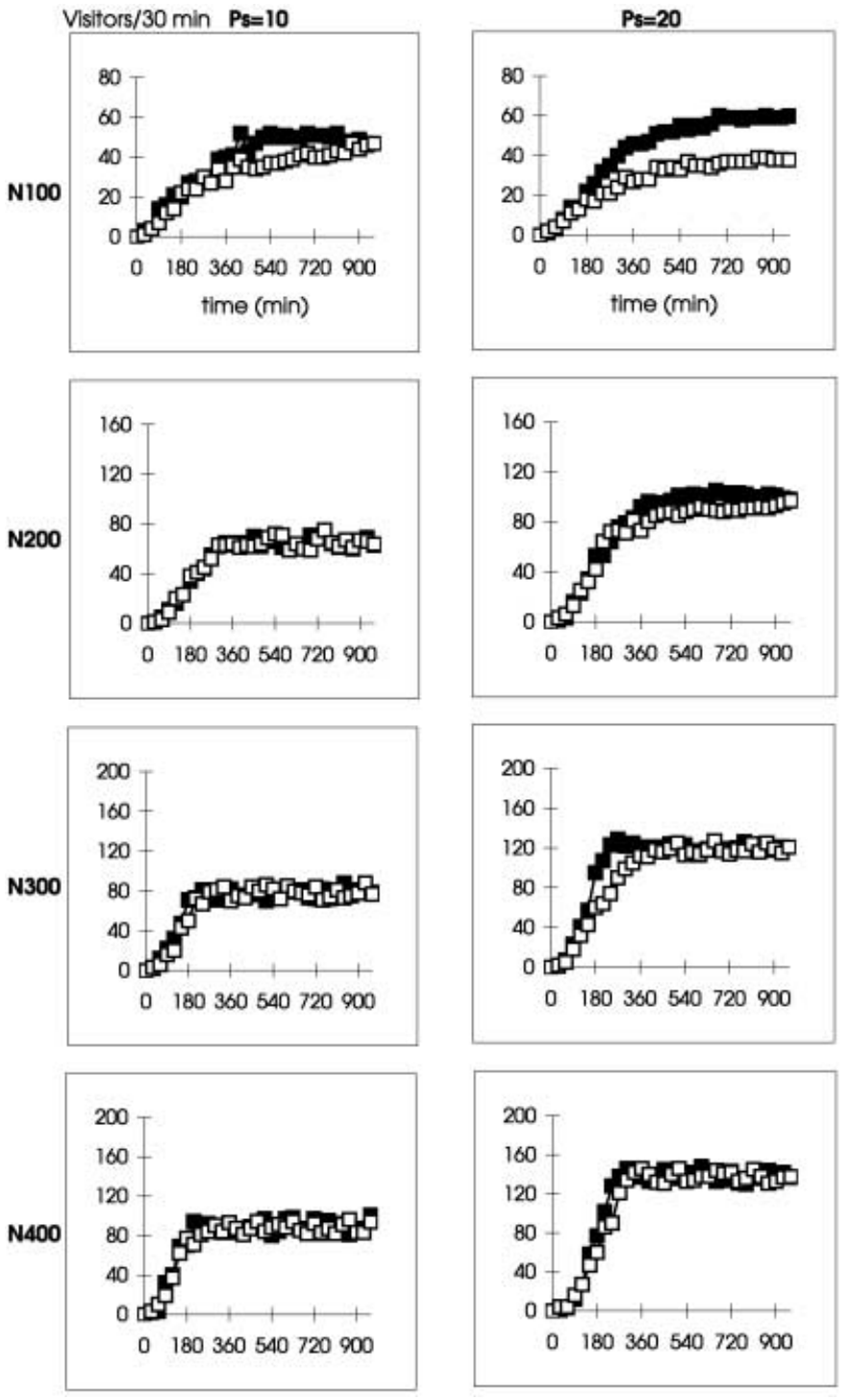

Fig. 2 Results of simulation runs showing when symmetry breaking emerges. Sixteen runs of 960 min each, showing the patterns of foragers visiting 2 nectar sources at $400 \mathrm{~m}$ north and south from the nest, with different sizes of the workforce $(N=100,200,300$, $400)$ and different patch sizes for both sources $(P s=10,20,40,60)$. Each dot represents the number of different foragers visiting a source during the previous half-hour. The sugar concentration for both sources stays constant at $2.0 \mathrm{M}$. The number of bees initially committed to each source is 10 . Other parameters are set to standard values

sugar concentration of both sources is set to a constant $2.0 \mathrm{~mol} / \mathrm{l}$.

Figure 2 shows the forager patterns for $N=100-400$ and patch size $P_{s}=10-60$. Each run started with ten bees initially committed to each source. For small patch sizes, symmetry breaking does not or hardly ever occurs. When the patch size of both sources is 20 , symmetry breaking occurs for $N=100$ bees. With larger patch sizes (Ps=40), symmetry breaking occurs for $N=100-200$, and with still larger patch sizes $(\mathrm{Ps}=60)$, it occurs for $N=100-300$. Overall, we see that emergence of symme-
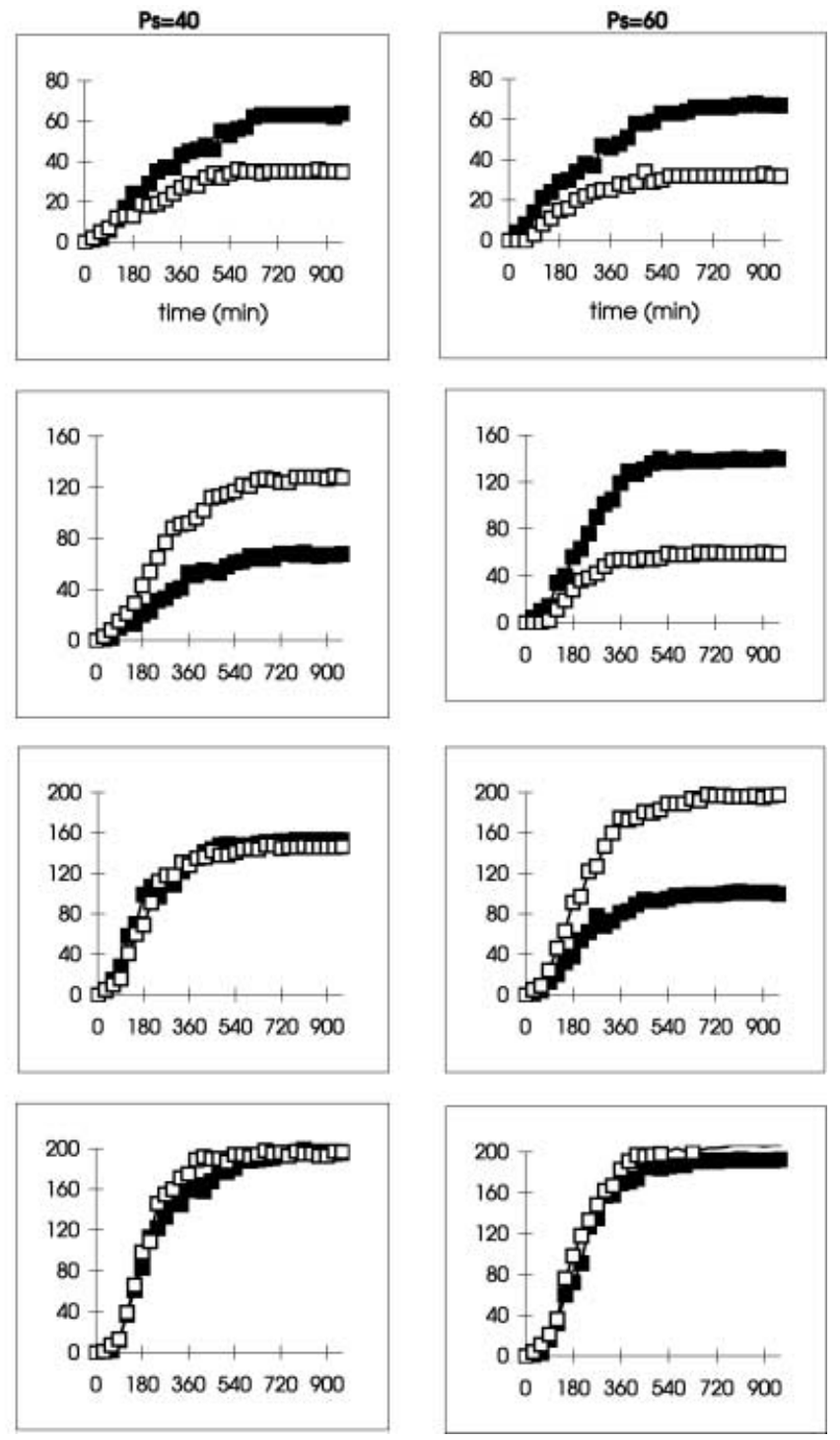

try breaking is more likely when the patch size of the two sources is rather large and when the pool of potential foragers is rather small.

To obtain an impression of the possible variation in symmetry breaking with respect to aspects such as time of onset and maximum asymmetry reached, we made 20 runs with the same initial conditions $(N=200$ and patch size $=40$ ), but with different initial random seeds for the random number generator. It seems that there is quite a variation in time of onset of asymmetry, varying between $t=90$ and $t=480 \mathrm{~min}$. Also, the variation in the maximum asymmetry found (i.e. the maximum absolute difference between the 2 numbers of foragers visiting both sources) is quite great; it ranges between 14 and 125 visitors. The total amount of energetic gain harvested by all bees together in these 20 runs ranges between $10,554 \mathrm{~kJ}$ and $11,790 \mathrm{~kJ}$; there appears to be no correlation between strength of asymmetry and harvest $(r=0.21)$. In these runs, ten bees were initially committed to each of two sources. We made 20 similar runs but now with 30 bees initially committed to each source. The results were 


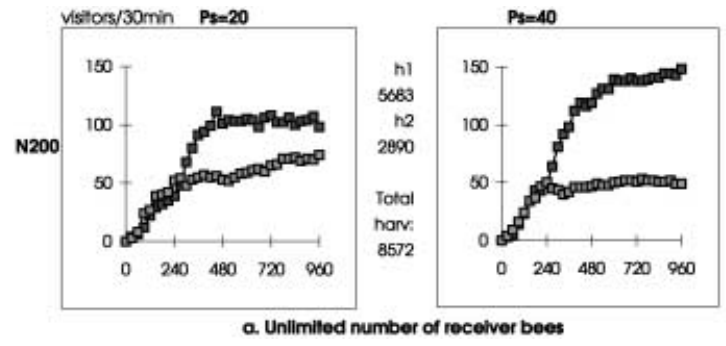

Fig. 3 Results of simulation runs showing the patterns of foragers visiting 2 nectar sources at $400 \mathrm{~m}$ north and south from the nest for the situation that there is an unlimited number of receiver bees (2 graphs at the left) (a) and for the situation that there is a limited number of 50 receiver bees and recruitment of additional receiver bees is not possible (2 graphs at the right) (b). Each dot represents the number of different foragers visiting a source during the previous half-hour. The sugar concentrations of both sources are initially set to $1.25 \mathrm{M}$. At time $=240 \mathrm{~min}$, the sugar concentration of source 1 (black dots) is set to $2.5 \mathrm{M}$, while the other one remains $1.25 \mathrm{M}$. The number of bees initially committed to each source is 30 . The forager work force $N$ is set to 200 for all runs; patch size, $P s$, is set to 20 or 40 . Other parameters are set to standard values

comparable: asymmetry ranges between 11 and 78 visitors; harvest ranges between 11,834 and $12,625 \mathrm{~kJ}$ (somewhat larger than the earlier runs since the higher numbers of initially committed bees lead to more bees starting to forage early on). For these runs also no significant correlation between strength of asymmetry and harvest was found $(r=-0.19)$. From this we may conclude that the occurrence of symmetry breaking appears not to be of any adaptive value for the colony. Whether symmetry breaking can have an adaptive value in real bee colonies remains to be investigated.

\section{Cross inhibition}

If the sugar concentration of one of two energetically equal nectar sources is suddenly increased, the number of foragers exploiting the other one will sometimes decrease. This phenomenon of cross inhibition between forager groups has been observed by Seeley (1995, pp 142-145), and is due to an inhibitory effect on the amount of dancing by bees exploiting the less valuable source, because of the longer search times for nectarreceiving bees upon entering the hive with a nectar load.

We made several simulation runs to compare the resulting forager patterns and colony harvests obtained for three situations. First, we simulated the situation in which the total number of available receiver bees is very high so that foragers do not experience any search time and also, therefore, no tremble dancing will occur nor will foragers abandon their source due to a long search time. Second, we investigated the situation in which there is a low and fixed number of potentially available receiver bees. In this situation, no recruitment of extra receiver bees by means of the tremble dance is allowed to take place. The actual number of idle receiver bees

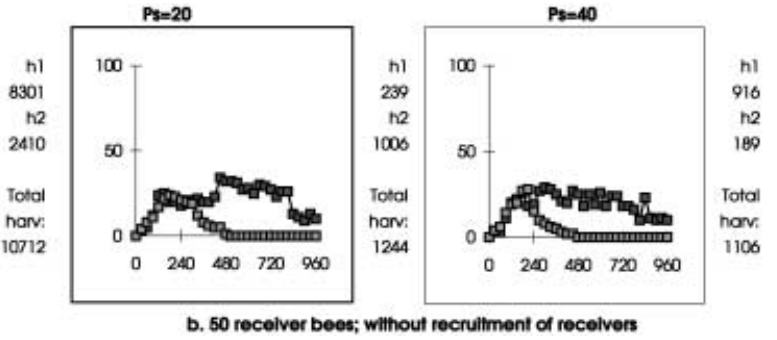

can still vary depending on the number of forager bees that are actually transferring their load, since during transference a forager bee occupies a receiver bee. As soon as the transfer is finished, the receiver bee becomes idle again. (The time it takes the receiver bee to store the nectar is not implemented in the current model.) The third situation is when recruiting of extra receiver bees is possible. In this last situation, we were interested in two different cases. One is when the probability of waggle dancing and of abandoning depends on the quality of the food (as described in the methods section), and the other case is when these probabilities do not differ for foragers bringing in different-quality foods. (In this last case, these probabilities were set to the values for a forager that has visited a rich 2.5-M sugar source.)

In the situation with an unlimited number of receiver bees, no cross inhibition took place. Figure 3 a shows two resulting forager patterns, one for the sources having a patch size of 20 and the other of 40; in both runs the forager pool size, $N$, was 200 . The numbers of foragers visiting the two sources keeps increasing until the patch size limits these numbers. For other patch sizes and other values of $N$, more or less similar patterns were found.

In the situation with a limited number of 50 receivers and where no receiver recruitment is possible, the number of visiting foragers starts decreasing for both sources when the numbers of these foragers have become about 25 (see Fig. 3b). Due to the long search times for an idle receiver bee, many returning foragers hardly waggle dance and will abandon their source. Obviously, the lack of available receiver bees is detrimental to the harvesting of nectar at both sources.

Next, we simulated the situation with initially 50 receiver bees and where the forager bees could recruit additional receivers by means of the tremble dance. When we made the probability of waggle dancing and of abandoning dependent on the quality of the nectar found by the forager, the phenomenon of cross inhibition emerged; that is, after time $=240$, when the sugar concentration of source 1 was increased, the number of foragers visiting the other source decreased (see Fig. 4, columns 1 and 2). Some of the forager patterns we found were quite similar to the forager patterns observed by Seeley (1995; Fig. 5.35 a, b, reproduced here in Fig. 4). This cross inhibition phenomenon seems quite functional, since by abandoning the poor source when another source becomes more profitable, more foragers are available for exploiting this rich source than when the poor-source exploiters remain with this source. To find out whether 

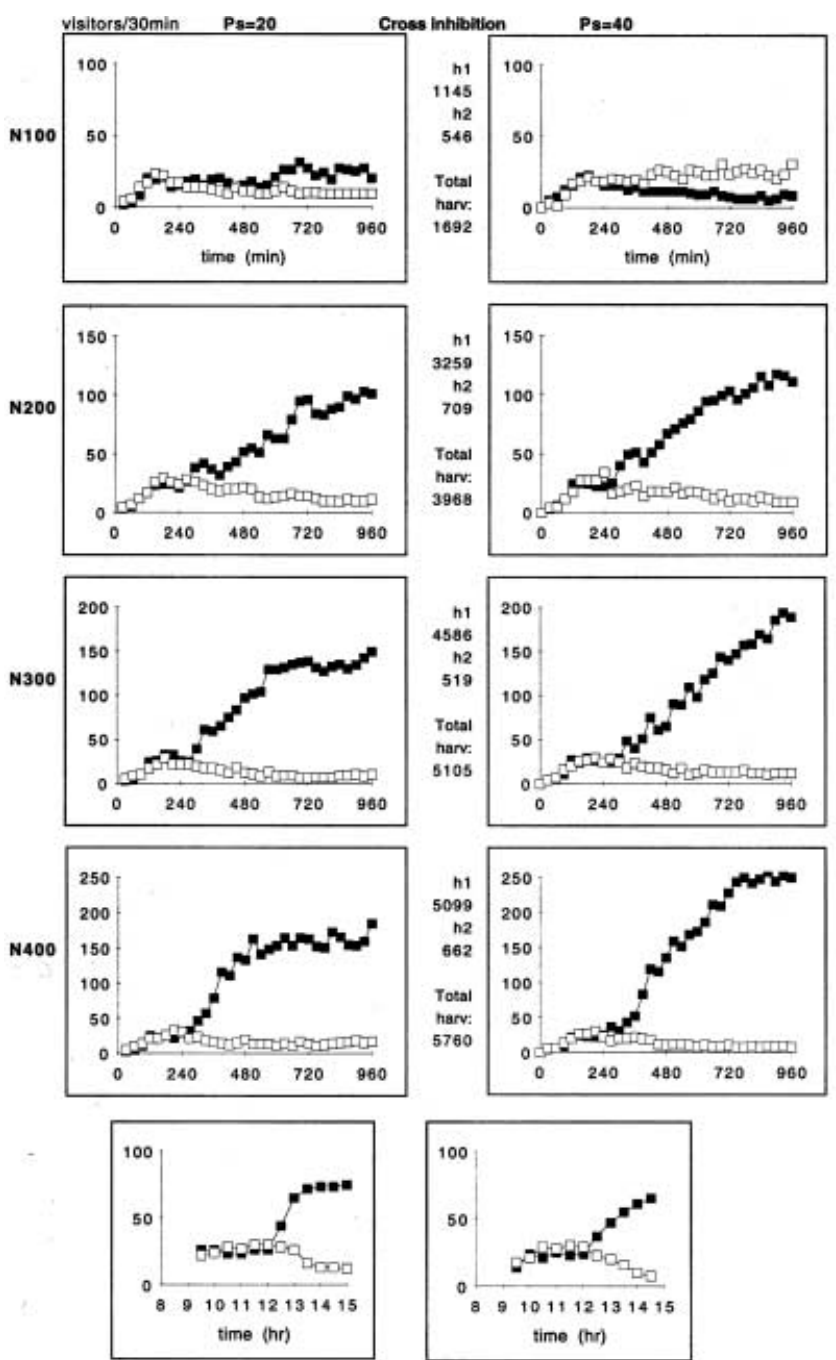

from: Soeley (1995, Fig. 5.35a)

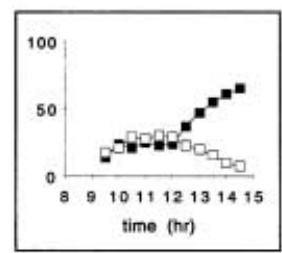

from: Soeiey (1905, Fig. 5.35b)

Fig. 4 Results of simulation runs showing when cross inhibition emerges. Two times eight runs of $960 \mathrm{~min}$ each, showing the patterns of foragers visiting 2 nectar sources at $400 \mathrm{~m}$ north and south from the nest, with different sizes of the workforce $(N=100,200$, $300,400)$ and different patch sizes for both sources $(P s=20,40)$. Each dot represents the number of different foragers visiting a source during the previous half-hour. The sugar concentrations of both sources are initially set to $1.25 \mathrm{M}$. At time $=240 \mathrm{~min}$, the sugar concentration of source 1 (black dots) is set to $2.5 \mathrm{M}$, while the other one remains $1.25 \mathrm{M}$. The number of bees initially committed to each source is 30 . Other parameters are set to standard values. Cross inhibition occurs for the eight runs at the left-hand side of the figure. Here the probability of abandoning the source, as well as the probability of waggle dancing, depends on the time till the forager bee finds a receiver bee, as well as on the quality of the nectar transferred. For the eight runs on the right-hand side the probabilities of these two subsequent behaviours also depend on the time till a receiver bee is found but not on the quality of the nectar brought in. In these runs, cross inhibition does not arise. For each run the harvests for both sources $(h 1, h 2)$, as well as the total harvest (in $\mathrm{kJ}$ ), is shown right of the graph. At the bottom of the figure are two graphs representing the forager patterns observed by Seeley (1995) during his experiment, in which the concentration of one source is increased from 1.25 to $2.5 \mathrm{M}$ at $12 \mathrm{~h}$, while the other one stays constant at $1.25 \mathrm{M}$
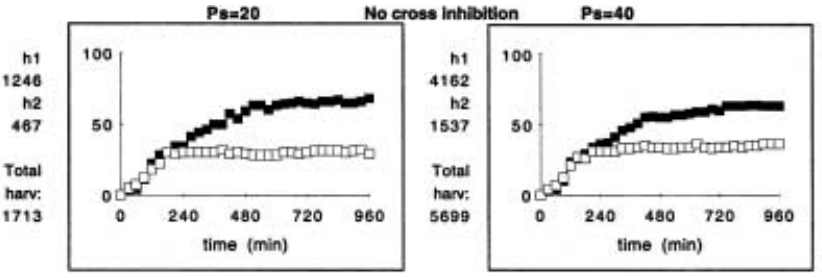

\section{$h 1$
3985
$h 2$ \\ $\mathrm{h} 2$
1726 \\ Total \\ harv:}
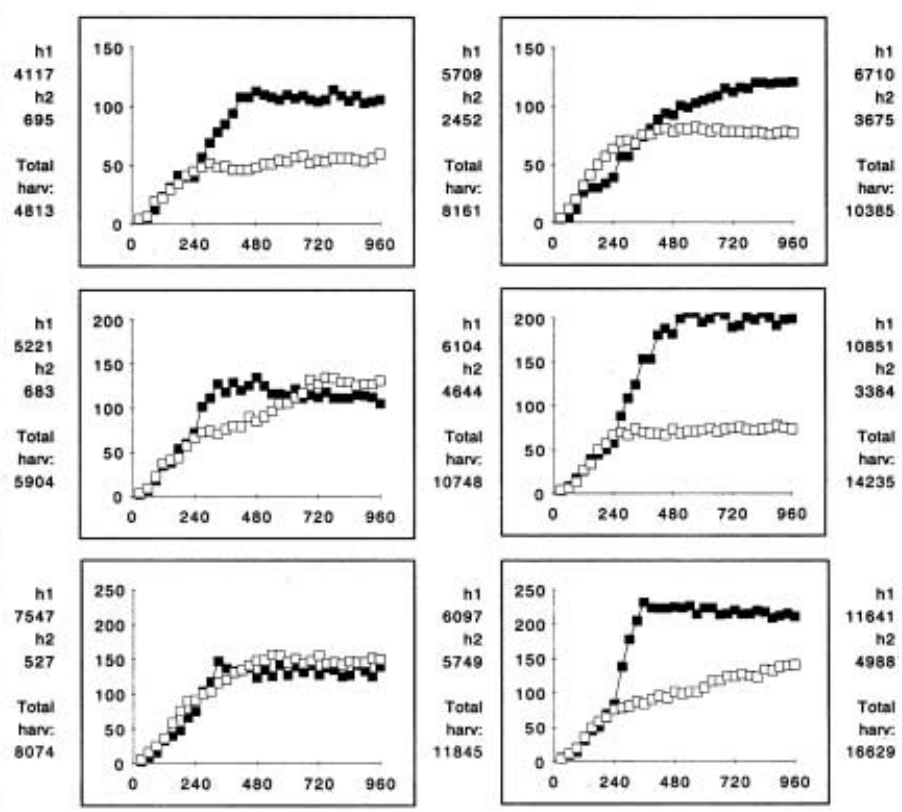

cross inhibition indeed resulted in a greater harvest, we compared it with a situation where no cross inhibition arises. We simulated the situation with initially 50 receiver bees and where recruitment of additional receivers is possible, but the probability of abandoning the source and of waggle dancing depends only on the time till a receiver bee is found and not on the sugar concentration of the transferred nectar. The resulting forager patterns for this situation are presented in Fig. 4 (columns 3 and 4). Remarkably, it appears that in all cases the harvest in the cross-inhibition situation (Fig. 4, columns 1 and 2) is smaller than in the situation without cross inhibition. These results show that when cross inhibition emerges, and thus a more differentiated allocation of the bees to the two sources is realized, this will not necessarily result in a greater harvest.

\section{Equal harvest-rate distribution}

The main reason why we introduced the possibility of varying the patch sizes of the nectar sources was to investigate under what circumstances the foragers of a colony would distribute themselves among the available sources such that the bees foraging on either source will bring in, on average, the same amount of energy (nectar) per time unit. We refer to this as the equal harvest-rate distribution. This investigation was inspired by a paper by Bartholdi et al. (1993), who presented a system of 
differential equations that models the allocation process in a hypothetical colony of bees, whose behaviour closely approximates the observed behaviour of real bees. The allocation pattern they found was one in which each forager experiences the same rate of "value" accumulation regardless of which patch she is visiting.

The authors pointed out that their "mathematical analysis does not rely on any assumption about what currency the bees are trying to maximize in their foraging, either at the individual or at the colony level" (p 35). Instead, they showed that when considering the steady state of a system of differential equations in which the average rates of recruitment and diversion (abandonment) are balanced, a measure of patch quality (denoted by $v_{x}$ in their paper, with $x$ indicating patch 1 or patch 2 ) arises, which leaves the precise currency of patch quality unspecified. This patch-quality measure produced by their model is a global type of measure: it is the average dance duration per trip for source $x\left(g_{x}\right)$ divided by the average diversion rate for source $x\left(f_{x}\right)$ (averaged over all bees foraging at patch $x$ ) as proportion of the average diversion rate for both sources $(f)$ (averaged over all bees foraging at patch 1 or 2): that is $v_{x}=g_{x} /\left(f_{x} / f\right)$. This patchquality measure leaves unanswered the question of which currency is actually used by the bees in regulating their behaviour.

In contrast, in our individual-oriented modelling approach, we start with specifying the criterion of source profitability (either net energetic efficiency or net rate of energy gain) that determines the amount of waggle dancing and other behaviours, and we subsequently examine the outcomes of our simulation runs to see when an equal harvest-rate distribution emerges. Another difference between our simulation model and the differential equations model of Bartholdi et al. is that, in our model, the precise dependency of dance duration on the source profitability, as well as the dependency of the abandoning probability on the source profitability, is included, whereas in the differential equations these dependencies are left unspecified and not taken into account. Yet another difference is that they do not include in their equations the possibility of error during the transfer of information when a bee attends a waggle dance. Nor do their differential equations take into account that outgoing bees recruited by a waggle dancer do not always find the advertised source. As will be shown here, inclusion of these so-called "lost recruits" in our model is essential if the foraging processes are to give rise to an equal harvest-rate distribution (EHD).

We began by taking net energy efficiency, (gaincost)/cost, as the forager's criterion of source profitability. From the results of an experiment to analyse the bee's criterion of nectar-source profitability, Seeley (1994) concluded that this criterion should be energetic efficiency. We used this criterion in our model to regulate the dance duration for bees that returned from a successful foraging trip. We were interested to see whether our individual-oriented simulation model would generate an EHD of foragers when we included the possibility of an inhibitory effect on the foragers exploiting a source, by limiting the number of bees that can simultaneously load nectar from this source. An EHD of foragers among the food patches containing different concentrations of sugar occurs when the mean per capita harvest rate for the two different patches are the same. In our simulations, source 1 was given a constant sugar concentration of $2.5 \mathrm{M}$ and source 2 a constant $1.5 \mathrm{M}$. Rather surprisingly, we did not find an EHD, when we calculated the average per capita rate of energy harvest for only those bees that were actually harvesting sugar from a source. Therefore, we decided to base this calculation not only on the bees that are actually exploiting the sources, but also on the bees that are recruited for a source but were not successful in finding it. Although these so-called lost recruits do not participate in the actual harvest, they are surely part of the colony's exploitation effort. The mean amount of energy harvested per half-hour per bee (comprising both the exploiters and the lost recruits) now appeared to be the same for the two sources, at least for some settings of the patch sizes, but clearly not for all settings. For some settings, for which we clearly expected an EHD to emerge, a significant difference between the average harvest rates for the two sources was still found. For instance, when the 2 patches had a patch size of 1 or 5 , and there were 300 potential foragers available, an EHD did not emerge.

Next we made a series of runs in which the bees use the net gain rate, (gain-cost)/time, instead of the energetic efficiency as profitability criterion. With this criterion regulating the forager's dance duration, an EHD emerged in exactly those situations that we expected. In Fig. 5, we present the pattern of visitors+lost recruits across time (left diagram) and the per capita nectar harvest rate across time (right diagram) for patch sizes of 5, 10 and 20 , and for the forager pool size varying between 100 and 500. We checked whether an EHD emerged by performing a paired $t$-test on the pairs of per capita harvest rates obtained for the last 17 half-hours starting at

Fig. 5 Results of simulation runs showing when an equal harvestrate distribution of bees emerges. Fifteen runs of 960 min each, showing the patterns of the summed numbers of different visitors and lost recruits visiting or looking for 2 nectar sources (source 1: black diamonds; source 2: white diamonds) at $400 \mathrm{~m}$ north and south from the nest, with different sizes of the workforce $(N=100-500)$ and different patch sizes for both nectar sources $(P s=5,10,20)$. The sugar concentration of source 1 is $2.5 \mathrm{M}$ throughout, while the concentration of source 2 is $1.5 \mathrm{M}$ throughout. As criterion of source profitability, the bees use the net gain rate, (gain-cost)/time. The number of bees initially committed to each source is 10 . Other parameters are set to standard values. For each run two graphs are shown: at the left-hand side the sum of different visitors and lost recruits for each source is shown ((visitors+lost recruits)/30 min); at the right-hand side the average harvest per bee, which may be a visitor or lost recruit, is shown (harvest/bee/30 min expressed in $\mathrm{kJ}$ ). Right from this graph is shown the $P$-value of a paired $t$-test comparing the average per capita harvests of the 2 sources for the last 17 half-hours (time $=480-960$ ). A non-significant $P$-value indicates the presence of an EHD. For the runs with $N=500$ the numbers of visitors only are also presented graphically (source 1: black squares; source 2: white squares) 


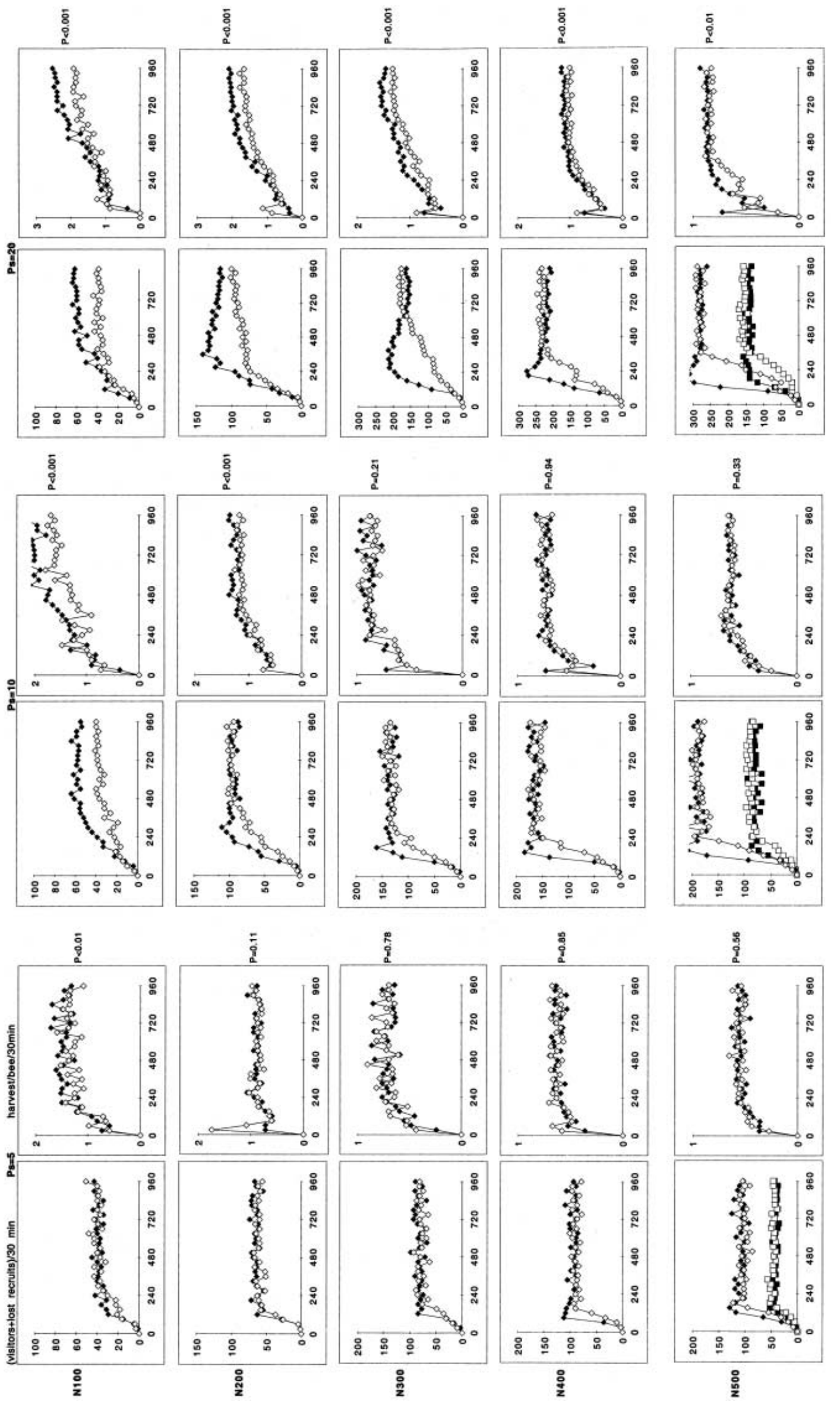


time $=480$. A non-significant difference indicates an EHD.

Looking at the 5 runs in which patch size was set to 5 (Fig. 5), we note that for $N=200$ or more an EHD arises, since the $t$-tests yield non-significant $P$-values $(P=0.11$, $0.78,0.85,0.56$, respectively). With a forager pool of only 100 bees, the average per capita harvest rate of the last 17 half-hours for the first source is significantly larger than for the second source. So, if there are not many forager bees, an EHD does not emerge. This is because, in this case, the number of bees visiting each source is limited by the small size of the forager pool. Since all available bees are busy with travelling between the nest and a source (the employed foragers) or with searching for a source (recruited bees) or with unloading and dancing, an equilibrium is reached when, for both sources, there are about 40 visitors+lost recruits (see Fig. 5, Ps=5 and $N=100$, left diagram). The equilibrium that is reached for larger forager pool sizes ( $N=200$ or more) is not due to the limitation set by the forager pool size but to the limitation set by the patch size. With a patch size of 5 , the maximum number of different foragers visiting each of the 2 sources in half an hour is about 30 when $N=100$. This number only slightly increases with higher $N$; if $N=500$ there are still only about 45 different bees visiting each source (Fig. 5, bottom left diagram, black squares: number of visitors on source 1, white squares: source 2). Of course, the number of lost recruits does increase with increasing $N$, resulting in a decreasing average per capita harvest rate with increasing $N$. The same pattern is visible in the 5 runs in which patch size has been set to 10 . For $N=100$ and 200, the numbers of visitors and lost recruits are limited by the size of the forager pool. For $N=300,400$ and 500, an EHD emerges because now the number of visitors at a source is limited by the patch size of this source. For patch sizes of 20 or 40 (runs with Ps $=40$ are not shown), an EHD does not arise since the equilibrium due to the limitation set by the forager pool size is already reached even when $N=500$.

All in all, we see that with a large patch size, an EHD does not arise and that an EHD only emerges when the forager pool size is large enough. This shows that it is indeed the negative feedback produced by the longer waiting times at smaller food sources that is responsible for the occurrence of an EHD of foragers among patches. In order to understand in more detail how an EHD arises, we will describe the temporal succession of numbers of different foragers visiting the sources and also of the numbers of different bees that are visitors and/or lost recruits. The pattern of this sequence can be understood as the result of negative and positive feedback processes. For 3 runs $(N=500 ; \mathrm{Ps}=5,10,20)$, we also present the numbers of different visitors in the same graph (Fig. 5, the black and white squares in the bottom row graphs). Looking at the graph for $\mathrm{Ps}=10$, we see that initially the number of visitors rises. For the richer source (source 1; $2.5 \mathrm{M}$; black squares), the increase is faster than for the poorer one (source $2 ; 1.5 \mathrm{M}$; white squares). This is due to the positive feedback processes involving the recruit- ment of bees through the waggle dances for both sources. From time $=180$, the number of visitors of source 1 levels off; for source 2, this occurs at time $=240$. Concurrently, the numbers of lost recruits (=difference between diamonds and squares) also increase and level off in a similar fashion. This levelling off is a consequence of the negative feedback process due to the limited size of the patch. Because the patch size is not unlimited, some bees have to fly around and wait for a place at the source to become free. This results in lower net gain rate for these bees (since their cost is higher and trip time is longer than for the bees that do not have to wait), which will lead afterwards to a shorter waggle dance. Some waiting bees flying around near the source will not succeed in acquiring a free place before they have exceeded their maximum flying distance (which has been arbitrarily set to a value of $1,000 \mathrm{~m}$ ), and will therefore return home empty. When the positive feedback on the recruitment process executed by the successful visitors together with the negative feedback executed by the unsuccessful bees is at an equilibrium for both sources, an equal harvestrate distribution will result. The mediating factor here is the duration of the waggle dance, which is directly dependent on the net gain rate.

\section{Discussion}

Deneubourg et al. (1999) suggested that, at present, a greater emphasis should be put on experiments, rather than on further speculation and facile computations. We believe that individual-oriented modelling is pre-eminently a tool that allows and even promotes a close coupling between collecting empirical data and discovering adequate concepts and explanatory theories, while at the same time it makes possible educated speculation about real-life processes and behaviour. Furthermore, individual-oriented modelling is a tool for understanding how sets of concepts at two different levels are related to each other. Individual-level concepts such as search ability, transmission accuracy, the bee's criterion of nectar source profitability, etc. have nothing in common with concepts at the level of forager patterns, such as symmetry breaking, cross inhibition and equal harvest-rate distribution. Behavioural rules link the individual-level concepts to the actual behaviour performed. The behaviour performed by all individuals gives rise to positive and negative feedback processes, which then provide a basis for understanding the emergence of collective patterns.

\section{Symmetry breaking}

Our results show that symmetry breaking is possible in collective foraging by honeybee colonies under certain circumstances, often already in the initial phase of simultaneous exploitation of large food sources. Because in that situation few bees are fixed to each source and foragers experience no interference at the large sources, 
chance may give the work force of one source a recruitment boost compared to the other work force, which leads to asymmetrical use of identical food sources. Symmetry breaking is a common pattern observed in self-organized systems (Pasteels et al. 1987; Millor et al. 1999), but is it just an artifact or may it be beneficial to the colony? In our simulations, the amount of nectar harvested and the strength of the emerging asymmetry are uncorrelated, implying that symmetry breaking does not provide some benefit for the colony, at least in the situations covered by our model. In situations where (for example) defending food sources against other visitors plays a role, focussing on one source can be beneficial for the colony. It is remarkable that, for as yet, symmetry breaking in honeybee foraging has not been observed (Seeley and Levien, cited in Pasteels et al. 1987; Bonabeau et al. 1997). Empirical research on symmetry breaking in collective foraging of bees is planned by the second author.

Under natural circumstances, the parallel occurrence of new food sources is probably common as individual plants of many species tend to perform synchronized blooming. However, simultaneous discovery of several food patches will be rare and even slight differences in time of discovery will lead to allocation of foragers according to the discovery order of the food sources (Pasteels et al. 1987). On the one hand, allocation of a majority of the foragers to one of several equally rewarding food sources may facilitate monopolization of that food source (Johnson 1983; Nagamitsu and Inoue 1997), and so might lead to a higher harvest than a more even distribution of foragers. However, interference at the food source increases with the number of foragers and may eventually lead to a decrease in the per capita harvest rate (see results section and discussion on EHD).

\section{Cross inhibition}

Our simulation results confirm the conclusion of Seeley (1995) that cross inhibition is caused by the increase in search time to find a nectar receiver. When there is an unlimited number of receiver bees and the returning forager bees do not have to search for a receiver, no cross inhibition occurs. Also, when there is a limited number of receiver bees and recruitment of additional receivers by means of the tremble dance is not possible, cross inhibition will not occur. In this last case, the number of foragers exploiting either source decreases. A necessary condition for cross inhibition to occur is that the probability of foragers to abandon their source after experiencing a long search time differs for different quality nectar brought in. If this abandoning probability does not depend on the quality of the nectar, the number of foragers visiting the poor source does not decrease.

When cross inhibition occurs, the colony concentrates relatively more of its exploitation effort on the most profitable food source. This does not lead, however, to a greater harvest as well (see
Fig. 4), which suggests that cross inhibition does not have an adaptive significance.

\section{When does an EHD emerge?}

An EHD emerges only if individuals used gain rate as currency and if lost recruits were incorporated in our calculations of the per capita harvest rate. The processes through which an EHD is reached include indirect interference at the food source (as was suggested by Bartholdi et al. 1993), which subsequently determines a nectar forager's abandoning probability and its probability of waggle dancing and, thus, the relative intensity of recruitment to the various food patches. For an EHD to emerge, food patches should not be too large and the number of foragers should be high enough, such that foragers interfere with each other indirectly at the source. It is noteworthy that in the equilibrium situation of an EHD, the harvest rates of individual bees differ substantially from each other but that the average per capita harvest rate is the same for both sources. This means that an EHD is not identical to an Ideal Free Distribution (IFD), in which individuals foraging on different-quality sources all achieve equal gain rates (Fretwell 1972). This, of course, follows directly from the assumptions on which the IFD is based, namely, that the animals are "ideal", meaning that they go to the patch where their intake rate is highest, and "free" in that they are able to enter any patch on an equal basis with the others already present. Both assumptions do not hold for a bee colony; individual bees lack the necessary global knowledge to choose the best patch, and when all places in a patch are occupied, newly arriving bees have to wait for a place to become free. So, although for individual bees an EHD is certainly not optimal, for the colony it is, since when this equilibrium has emerged, a reallocation of bees to the sources will only result in a smaller harvest for the colony.

The question of whether foragers use gain rate or net energetic efficiency as a currency to assess nectar source profitability remains unresolved. Re-analysis of Seeley's (1994) data shows that both currencies may have been used by his bees (see Appendix). In his discussion, Seeley concludes that bees may use each of these currencies in different situations. Surprisingly, we only obtain an EHD if individual bees use gain rate as currency. Further empirical studies should clarify which currency bees use.

Honeybee collective intelligence and food source ecology

Another conclusion from our simulation studies is that the size of food patches is an essential factor determining which foraging patterns will emerge: symmetry breaking and cross inhibition occur only at large patches, whereas an EHD emerges only at smaller patches $(\mathrm{Ps}=10)$. Patch size is generally not considered to be an important factor 
in honeybee collective foraging, and many studies use artificial food sources that provide space for about 20 bees at any one time (reviewed in von Frisch 1967; Seeley 1995). Our parameter patch size can be interpreted ecologically as reflecting the number of flowers that can be exploited simultaneously at a patch. In our model and in most empirical studies, patch size remains the same over time, whereas under natural circumstances patch size decreases over time, because the number of empty flowers increases as a result of foraging (except in the unlikely event that nectar secretion or opening of new flowers occurs at the same rate as nectar collecting). This suggests that in natural foraging, symmetry breaking and cross inhibition may occur during the initial phase of nectar source exploitation when patch size is large and interference at the food source relatively unimportant. Although an EHD is the optimal distribution of foragers, it will not often be reached in nature. This is because patch size is probably not constant but decreases with number of visits to the patch. Therefore, interference at the food source is too low in the initial phase of exploitation of large food patches. Once exploitation of the food source progresses, the EHD to be reached changes continuously, from the moment the proportion of empty flowers reaches the threshold for interference till the patch is emptied.

Although the emergence of an EHD and symmetry breaking may be rare events in the honeybee's natural foraging ecology and may be restricted to experimental situations, they allowed us to improve our simulation model such that we now have a more general model which may explain more natural foraging patterns and predict both new collective foraging patterns and behavioural rules essential to our understanding of the honeybee's collective intelligence.

Acknowledgements We are very grateful to Lada Lhotka for allowing us to use his programming environment for individual-oriented modelling HOBO. This work was supported by the Netherlands Foundation for the Advancement of Tropical Research (NWO-WOTRO) grant W84-471 to M.J. Sommeijer. We thank two anonymous referees for their comments on the paper.

\section{Appendix}

Since experimenting with our model led us to consider gain rate to be the most probable source profitability criterion, we took a closer look at the experimental results upon which Seeley (1994) based his conclusion that it is energetic efficiency and not gain rate that is used by the bees as profitability criterion. In his Table 1, he presents for each of two trials, two values of (gain-cost)/time; for the first trial, 1.43 and 1.61, and for the second, 1.83 and 1.99. Since their ratios of 0.88 and 0.92 , respectively, are less than the ratios found for (gain-cost)/cost (1.00 and 0.98 ), this last criterion seems the most appropriate. However, a critical factor, upon which the calculations leading to these values depend, is the estimated values of the concentration of sucrose solutions for the near feeder that would elicit dances with the same mean number of waggle runs as in the dances for the far feeder. These values have been obtained by linearly interpolating between the results from the trials in the morning and the afternoon. In the morning, the experiments were done with the far $(550 \mathrm{~m})$ feeder filled with a $2.50 \mathrm{~mol} / \mathrm{l} \mathrm{su}$ crose solution, and the near $(250 \mathrm{~m})$ feeder filled with a $1.75 \mathrm{~mol} / \mathrm{l}$ sucrose solution. In the afternoon, for both trials, the far feeder was left at $2.50 \mathrm{~mol} / \mathrm{l}$ and the near feeder was switched to $1.25 \mathrm{~mol} / \mathrm{l}$. Based on the ratios of the two waggle runs/trip values, the sucrose solution for equal dancing was estimated by linear interpolation to be 1.32 and 1.46 for the two trials, respectively. One could also ask, however, what the sucrose solution of the near feeder would have to be for the gain rates to be equal for the two sources. It is quite easy to calculate this: for the first trial, the sucrose solution of the near feeder would have to be 1.48 to arrive at a (gain-cost)/time of 1.61 (all other things being equal). For the second trial, the sucrose solution of the near feeder would have to be 1.59 to arrive at a (gain-cost)/time of 1.99 , the gain rate for the far feeder. In fact, the value of the sucrose solution obtained for the first trial is quite close to the actual solution of $1.5 \mathrm{~mol} / \mathrm{l}$ used in the test trial (of $16 \mathrm{July}$ ), which is used for calculating the values of the three possible criteria. Unfortunately, the values of the waggle runs/trip for this trial have not been presented, making checking of the equality of these values for the near and far feeder impossible. Since these values of the sucrose solutions are not very far off from the values estimated by linear interpolation, it seems that the results of this experiment are not fully conclusive in rejecting gain rate as a possible criterion of profitability. Interestingly, in the discussion of his paper, Seeley (1994) suggests that it may well be that nectar-foraging bees maximize either energetic efficiency or the rate of energy acquisition, depending on the colony's energy situation.

\section{References}

Anderson C (1998) Simulation of the feedbacks and regulation of recruitment dancing in honeybees. Adv Complex Syst $1: 267-282$

Anderson C, Ratnieks FLW (1999) Worker allocation in insect societies: coordination of nectar foragers and nectar receivers in honeybee (Apis mellifera) colonies. Behav Ecol Sociobiol 46:73-81

Bartholdi JJ, Seeley TD, Tovey GA, Vate JH van de (1993) The pattern and effectiveness of forager allocation among flower patches by honeybee colonies. J Theor Biol 160:23-40

Biesmeijer JC, Vries H de (2001) Exploring and exploiting of food sources by social insect colonies: a revision of the scoutrecruit concept. Behav Ecol Sociobiol 49:89-99

Bonabeau E, Theraulaz G, Deneubourg J-L, Aron S, Camazine S (1997) Self-organization in social insects. Trends Ecol Evol 12:188-193

Camazine S, Sneyd J (1991) A model of collective nectar source selection by honey bees: self- organization through simple rules. J Theor Biol 149:547-571

Camazine S, Deneubourg JL, Franks NR, Sneyd J, Theraulaz G, Bonabeau E (2001) Self-organization in biological systems. Princeton University Press, Princeton 
Deneubourg JL, Camazine S, Detrain S (1999) Self-organization or individual complexity: a false dilemma or a true complementarity? In: Detrain C, Deneubourg JL, Pasteels JM (eds) Information processing in social insects. Birkhäuser, Basel, pp 401-408

Detrain C, Deneubourg JL, Pasteels JM (1999) Information processing in social insects. Birkhäuser, Basel

Fretwell SD (1972) Populations in a seasonal environment. Princeton University Press, Princeton

Frisch K von (1967) The dance language and orientation of bees. Harvard University Press, Cambridge

Hogeweg P, Hesper B (1990) Individual-oriented modelling in ecology. Math Comput Modell 13:83-90

Johnson LK (1983) Foraging strategies and the structure of stingless bee communities in Costa Rica. In: Jaisson P (ed) Social insects in the tropics, vol 2. Université du Paris-Nord, pp 32-58

Judd TM (1995) The waggle dance of the honeybee: which bees following a dancer successfully acquire the information? J Insect Behav 8:343-353

Judson OP (1994) The rise of the individual-based model in ecology. Trends Ecol Evol 9:9-14

Lhotka L (1994) Implementation of individual-oriented models in aquatic ecology. Ecol Modell 74:47-62

Millor J, Pham-Delegue M, Deneubourg JL, Camazine S (1999) Self-organized defensive behavior in honeybees. PNAS 96: 12611-12615

Moritz RFA, Kryger P (1994) Self-organization of circadian rhythms in groups of honeybees (Apis mellifera L.) Behav Ecol Sociobiol 34:211-215

Nagamitsu T, Inoue T (1997) Aggressive foraging of social bees as a mechanism of floral resource partitioning in an Asian tropical rainforest. Oecologia 110:432-439
Pasteels JM, Deneubourg J-L, Goss S (1987) Self-organization mechanisms in ant societies. I. Trail recruitment to newly discovered food sources. In: Pasteels JM, Deneubourg J-L (eds) From individual to collective behavior in social insects. Experientia Suppl 54:155-175

Seeley TD (1992) The tremble dance of the honeybee: message and meanings. Behav Ecol Sociobiol 31:375-383

Seeley TD (1994) Honey bee foragers as sensory units of their colonies. Behav Ecol Sociobiol 34:51-62

Seeley TD (1995) The wisdom of the hive. Harvard University Press, Cambridge

Seeley TD, Buhrman SC (1999) Group decision making in swarms of honey bees. Behav Ecol Sociobiol 45:19-31

Seeley TD, Tovey CA (1994) Why search time to find a foodstorer bee accurately indicates the relative rates of nectar collecting and nectar processing in honeybee colonies. Anim Behav 47:311-316

Seeley TD, Camazine S, Sneyd J (1991) Collective decision-making in honeybees: how colonies choose among nectar sources. Behav Ecol Sociobiol 28:277-290

Seeley TD, Kühnholz S, Weidenmüller A (1996) The honeybee's tremble dance stimulates additional bees to function as nectar receivers. Behav Ecol Sociobiol 39:419-427

Visscher PK, Camazine S (1999) The mystery of swarming honeybees: from individual behaviors to collective decisions. In: Detrain C, Deneubourg JL, Pasteels JM (eds) Information processing in social insects. Birkhäuser, Basel, pp 355-378

Vries H de, Biesmeijer JC (1998) Modelling collective foraging by means of individual behaviour rules in honey-bees. Behav Ecol Sociobiol 44:109-124

Watmough J, Camazine S (1995) Self-organized thermoregulation of honey bee clusters. J Theor Biol 176:391-402 